\title{
CHANNEL FEEDBACK QUANTIZATION METHODS FOR MISO AND MIMO SYSTEMS
}

\author{
June Chul Roh and Bhaskar D. Rao \\ Department of Electrical and Computer Engineering \\ University of California, San Diego \\ La Jolla, CA 92093-0407, USA \\ Email: jroh@ece.ucsd.edu, brao@ece.ucsd.edu
}

\begin{abstract}
In this paper, we investigate quantization of multiple antenna channel to feed back through a low-rate feedback channel. Specifically, for multiple-input single-output (MISO) systems we propose a new design criterion and the corresponding design algorithm for quantization of the random beamforming vector. For multiple-input multiple-output (MIMO) channels, which have multiple orthonormal vectors as channel spatial information for quantization, a matrix factorization method is proposed which provides a way to exploit the geometrical structure of orthonormality while quantizing the spatial information matrix. Results show that the quantization bit allocation over multiple spatial channels has a critical effect on the performance, and that the optimum bit allocation depends on the operating transmit power of the system.
\end{abstract}

Keywords - Multiple antennas, MIMO systems, channel state information, vector quantization, transmit beamforming.

\section{INTRODUCTION}

Communication systems using multiple antennas have attracted considerable attention because of the potential improvements in transmission rate and/or link performance. The capacity of a multiple antenna channel depends on the availability of channel state information (CSI) at the transmitter [1]. A higher channel capacity can be achieved when the transmitter has perfect knowledge of channel by using the channel information in transmission. However, in many practical systems, especially when the channel information is provided to the transmitter through a low-rate feedback channel, perfect CSI at the transmitter (CSIT) is not possible. Also perfect CSIT is almost impossible to be achieved in timevarying channels. In a general MIMO flat-fading setting, the feedback information is a beamforming (or precoding) matrix, usually an orthonormal column matrix, and the power allocation along the beams. There are a few studies dealing with how to feed back the channel information. Some researchers have worked on feedback of channel information in vector form, for example, for MISO channels [2], [3] and for the principal eigen-mode of MIMO channels [4]. Feedback of channel information in matrix form for MIMO channels have only recently begun to be addressed [5], [6].

For a MISO channel, the feedback information is a complex unit-norm beamforming vector. In this paper, we look at the problems as a vector quantization (VQ) problem. The conventional mean squared error (MSE) criterion, though convenient

This research was supported by CoRe grant No. 02-10109 sponsored by Ericsson. for quantization design, may not be suitable because the more appropriate criteria for effective communication is channel capacity or signal-to-noise ratio (SNR). We propose a new design criterion, namely, maximizing mean squared innerproduct (MSIP). A VQ design algorithm (modified Lloyd algorithm) for the MSIP criterion was developed. A general MIMO channel has multiple orthonormal vectors as channel spatial information for quantization. A good quantizer needs to exploit the geometrical structure of orthonormality while quantizing the matrix spatial information. Also the multiple spatial channels in MIMO channels have different contributions to the channel capacity because each spatial channel has a different channel gain. This also has to be taken into account while designing a quantizer, leading to a problem of bit allocation.

We use the following notations. $A^{\dagger}$ and $A^{T}$ indicate the conjugate transpose and the transpose of matrix $A$, respectively. $I_{n}$ is the $n \times n$ identity matrix and $0_{m, n}$ means the $m \times n$ zero matrix. $\operatorname{diag}\left(a_{1}, \ldots, a_{n}\right)$ is a square diagonal matrix with $a_{1}, \ldots, a_{n}$ along the diagonal. The inner product between two vectors is defined as $\langle u, v\rangle=u^{\dagger} v$ and the 2-norm of vector $v$ is denoted by $\|v\|=\langle v, v\rangle^{1 / 2}$. $E[\cdot]$ represents the expectation operator, and $\mathcal{C N}(\mu, \Sigma)$ is the complex Gaussian random vector with mean $\mu$ and covariance $\Sigma$. Uniform distribution over a set $S$ is denoted by $\mathcal{U}(S)$. An $m \times n(m \geq n)$ matrix $A$ with orthonormal columns, i.e., $A^{\dagger} A=I_{n}$, will be called orthonormal column matrix.

\section{SySTEM ModeL}

We consider a multiple antenna system with $t$ transmit and $r$ receive antennas. Assuming flat-fading, the MIMO channel is modeled by the channel matrix $H \in \mathbb{C}^{r \times t}$. That is, the channel input $x \in \mathbb{C}^{t}$ and the channel output $y \in \mathbb{C}^{r}$ have the following relationship:

$$
y=H x+\eta
$$

where $\eta$ is the additive white Gaussian noise vector distributed by $\mathcal{C N}\left(0_{r, 1}, I_{r}\right)$. We denote the rank of $H$ by $m$. And, the singular value decomposition (SVD) of $H$ is given by $H=$ $U_{H} \Sigma_{H} V_{H}^{\dagger}$, where $U_{H} \in \mathbb{C}^{r \times r}$ and $V_{H} \in \mathbb{C}^{t \times t}$ are unitary matrices and $\Sigma_{H} \in \mathbb{R}^{r \times t}$ contains the singular values $\sigma_{1} \geq$ $\ldots \geq \sigma_{m}>0$ of $H$. We impose a constraint on the transmit power by $E\left[x^{\dagger} x\right] \leq P_{T}$.

We assume that perfect CSI is known to the receiver. The transmit beamforming along the first principal eigenvectors of 
$H^{\dagger} H$ is the optimum choice in capacity sense [7]. Therefore, the first $n(1 \leq n \leq m)$ column vectors of $V_{H}$ are to be quantized and fed back to the transmitter as channel spatial information. We assume a finite low-rate feedback channel ( $B$ bits per channel update) without error and delay. For notational convenience, let us denote the $i$-th column vector of $V_{H}$ by $v_{i}$ and define the first $n$ column vectors of $V_{H}$ as $V$, i.e., $V=\left[v_{1}, \ldots, v_{n}\right]$. Also we define $\Sigma=\operatorname{diag}\left(\sigma_{1}, \ldots, \sigma_{n}\right)$, a submatrix of $\Sigma_{H}$.

The quantized matrix $\hat{V}=\mathcal{Q}(V)$ is employed as a transmit beamforming matrix. That is, an information-bearing symbol vector $s=\left[s_{1}, \ldots, s_{n}\right]^{T}$ is transmitted through $x=\hat{V} s$, resulting in the receive signal

$$
y=H \hat{V} s+\eta \text {. }
$$

Here we assume that $s \sim \mathcal{C N}\left(0_{n, 1}, \Phi\right)$ and $\Phi=P_{T}$. $\operatorname{diag}\left(\gamma_{1}, \ldots, \gamma_{n}\right)$ with $\gamma_{i} \geq 0$ and $\sum_{i} \gamma_{i}=1$. The vector $\gamma=$ $\left[\gamma_{1}, \ldots, \gamma_{n}\right]$ will be called power allocation information. With perfect knowledge of channel at the receiver, the optimum power allocation can be calculated at the receiver [8]. We will also consider quantizing and feeding back the power allocation information. When the transmitter has the feedback information $(\hat{V}, \hat{\gamma})$, the instantaneous capacity (when $n=m$ ) is given by

$$
C_{\hat{V}, \hat{\gamma}}(H)=\log \operatorname{det}\left[I_{n}+V^{\dagger} \hat{V} \Phi(\hat{\gamma}) V^{\dagger} V \Sigma^{2}\right]
$$

where $\Phi(\hat{\gamma})$ is the power allocation associated with $\hat{\gamma}$, i.e., $\Phi(\hat{\gamma})=P_{T} \cdot \operatorname{diag}(\hat{\gamma})$.

\section{QUANTIZATION OF RANDOM BEAMFORMING VECTOR}

In this section, we consider MISO channels with $t$ transmit antennas. The channel matrix is a row vector: let us denote $H=h^{\dagger}\left(h \in \mathbb{C}^{t}\right)$. The channel vector $h$ is also represented in its magnitude and direction as $h=\sigma v$, where $\sigma=\|h\|$ and $v=h /\|h\|$. It is well-known that transmit beamforming along $v$ maximizes the received SNR and also the mutual information. Before discussing quantization of the random unit-norm vector $v$, let us first find the probability distribution. It is also well-known that for random channel $h$ with i.i.d. $\mathcal{C N}(0,1)$ entries, $v$ is independent of $\sigma$ and, moreover, uniformly distributed over the unit-norm sphere $\mathcal{S}_{t}=\left\{u \in \mathbb{C}^{t}:\|u\|=1\right\}$.

Let us assume the transmitter uses a quantized version of $v$, $\hat{v}=\mathcal{Q}(v)(\|\hat{v}\|=1)$ as transmit beamforming vector. Then, the received signal $y$ is represented as

$$
y=\sigma\langle v, \hat{v}\rangle s+\eta
$$

where $s \in \mathbb{C}\left(E\left[|s|^{2}\right]=P_{T}\right)$ is the transmit symbol conveying information data. And the received SNR is simply

$$
\mathrm{SNR}=\sigma^{2}|\langle v, \hat{v}\rangle|^{2} P_{T} .
$$

Therefore, we can see that, for the i.i.d. channel, maximizing $E[\mathrm{SNR}]$ is equivalent to maximizing $E|\langle v, \hat{v}\rangle|^{2}$. This motivates us to consider a new design criterion for quantization of the directional vector $v$.

\section{A. The MSIP Criterion and VQ Design Algorithm}

In this section, we will discuss the quantization of the random beamforming vector and a new VQ design method will be introduced.

New Design Criterion: Design a quantizer to maximize the mean squared inner-product (MSIP), that is,

$$
\max _{\mathcal{Q}(\cdot)} E|\langle v, \mathcal{Q}(v)\rangle|^{2} .
$$

It would be instructive to compare the this criterion with the MSE criterion, which is common in quantization study. Since $v$ and $\hat{v}$ are both unit-norm, in the MSE criterion, $\min E\|v-\hat{v}\|^{2}$ is equivalent to $\max E[\operatorname{Re}\langle v, \hat{v}\rangle]$. Therefore, the two criterions are different. ${ }^{1}$ It turns out that the performance (average SNR and also mutual information) of the quantizer designed with MSIP criterion is superior to that with MSE criterion.

Design Algorithm: One of the virtues with the MSIP criterion is that it has a closed-form VQ design algorithm (modified Lloyd algorithm). The original Lloyd algorithm is based on two conditions: optimum encoder for a fixed decoder, and optimum decoder for a fixed encoder [9]. They are also called the nearest neighborhood condition (NNC) and the centroid condition (CC). The same approach is used here.

1. $N N C$ : For given code vectors $\left\{\hat{v}_{i} ; i=1, \ldots, N\right\}$, the optimum partition cells satisfy

$\mathcal{R}_{i}=\left\{v \in \mathcal{S}_{t}:\left|\left\langle v, \hat{v}_{i}\right\rangle\right| \geq\left|\left\langle v, \hat{v}_{j}\right\rangle\right|, \forall j \neq i\right\}$, for $i=1, \ldots, N$

where $\mathcal{R}_{i}$ is the partition cell (Voronio region) for the $i$ th code vector $\hat{v}_{i}$.

2. $C C$ : For a given partition $\left\{\mathcal{R}_{i} ; i=1, \ldots, N\right\}$, the optimum code vectors satisfy

$$
\hat{v}_{i}=\arg \max _{\hat{v} \in \mathcal{R}_{i}} E\left[|\langle v, \hat{v}\rangle|^{2} \mid v \in \mathcal{R}_{i}\right] \text {, for } i=1, \ldots, N .
$$

Since $E\left[|\langle v, \hat{v}\rangle|^{2} \mid v \in \mathcal{R}_{i}\right]=\hat{v}^{\dagger} E\left[v v^{\dagger} \mid v \in \mathcal{R}_{i}\right] \hat{v}$, the solution of the above optimization problem is

$$
\hat{v}_{i}=\text { (principal eigenvector) of } E\left[v v^{\dagger} \mid v \in \mathcal{R}_{i}\right] .
$$

The above two conditions are iterated until the MSIP converges. The MSIP method is similar to the VQ design method developed by Narula [2]. It turns out that the two methods perform differently in spatially correlated channels [10].

\section{B. Numerical Results and Comparison with Other Criteria}

We utilize the algorithm to design codebooks for different dimensions and different number of quantization bits $B\left(N=2^{B}\right)$. Here, channels with i.i.d. $\mathcal{C} \mathcal{N}(0,1)$ entries are considered. Fig. 1 shows an example for $t=3$ with different quantization bits. Note that when $B=\infty$ the MSIP $E|\langle v, \hat{v}\rangle|^{2}=1$. For comparison, two other design methods are considered: i) $\min \max _{i \neq j}\left|\left\langle\hat{v}_{i}, \hat{v}_{j}\right\rangle\right|$; and ii) $\min E\|v-\hat{v}\|^{2}$

\footnotetext{
${ }^{1}$ Note that the two criterions are same for the real vector case, but not for the complex vector case.
} 
(MSE criterion). The first method was studied in [3], [4]. Although it can be another reasonable design criterion, there is no close-form VQ design algorithm that can be employed in designing a codebook for arbitrary dimension and arbitrary codebook size. The codebooks compared in the figure are ones that are tabulated for low number of quantization bits $(B=$ $1,2,3)$ in [4]. Furthermore, the design approach developed is useful irrespective of the distribution of $v$, while the method i) is only good for the i.i.d. channel. The results show that the proposed MSIP method has a comparable performance (in terms of MSIP) with the method i), and performs better than the method ii).

The average capacity of MISO channel with quantized channel feedback is given by $C_{\mathcal{Q}(v)}=E[\log (1+$ $\left.\left.P_{T} \sigma^{2}|\langle v, \mathcal{Q}(v)\rangle|^{2}\right)\right]$. Numerical results when the codebooks designed with the MSIP criterion are shown in Fig. $2(t=3$ and $B=1, \ldots, 6)$. For comparison, the capacities for the two extreme cases of complete CSIT $\left(C_{\text {Full }}\right)$ and no CSIT $\left(C_{\text {None }}\right)$ are included in the figure. The comparative results for the two competing methods in terms of MSIP also hold for the capacity measure (results are not shown here).

A more general method for quantization of beamforming vector, especially for spatially correlated channels, and also performance analysis of systems with quantized beamforming can be found in [10]. In the i.i.d. channel, the method reduces to the MSIP method in this paper.

\section{EXTENSION TO MIMO CHANNELS}

In this section, we consider channel information quantization for MIMO channels with $t$ transmit and $r$ receive antennas. As mentioned in Section II, the channel spatial information we want to feed back is a $t \times n$ orthonormal column matrix $V=\left[v_{1}, \ldots, v_{n}\right](n \leq m \leq t)$. First, for ease of readability, we summarize the key results that lead to the proposed quantization method along with the method itself.

1) An orthonormal column matrix $V \in \mathbb{C}^{t \times n}$ can be uniquely represented by a set of unit-norm vectors with different dimensions, $q_{1} \in \mathcal{S}_{t}, q_{2} \in \mathcal{S}_{t-1}, \ldots$, $q_{n} \in \mathcal{S}_{t-n+1}$, where $\mathcal{S}_{t}=\left\{u \in \mathbb{C}^{t}:\|u\|=1\right\}$.

2) For random channel $H$ with i.i.d. $\mathcal{C} \mathcal{N}(0,1)$ entries, the each unit-norm vector is uniformly distributed over the corresponding unit-norm sphere, i.e., $q_{i} \sim \mathcal{U}\left(\mathcal{S}_{t-i+1}\right)$ for $i=1, \ldots, n$. Furthermore, the unit-norm vectors $q_{i}$ are statistically independent.

3) For $i=1, \ldots, n$, the unit-norm vector $q_{i}$ is quantized using a codebook $\mathcal{C}_{i}$ that is designed for random unitnorm vector in $\mathbb{C}^{t-i+1}$ with the MSIP criterion.

The above three items will be dealt with in the following three subsections.

\section{A. Vector-Form Parameterization of Spatial Information}

Let us start with a Lemma that will play an important role in vector-form parameterization of spatial information.

Lemma 1: Consider a unitary matrix $G_{1}$ whose first column is $v_{1}$, the first column of $V$, and the remainder columns are arbitrarily chosen to satisfy the unit-norm and the mutual orthogonality property. Let us consider multiplication $G_{1}^{\dagger} V$. Then, it has the form of

$$
G_{1}^{\dagger} V=\left[\begin{array}{cc}
1 & 0 \\
0 & V^{(2)}
\end{array}\right]
$$

where $V^{(2)}$ is a $(t-1) \times(n-1)$ orthonormal column matrix and 0's are zero matrices with appropriate sizes.

Proof: Denote $G_{1}=\left[v_{1} \mid B\right]$, where $B$ is a $t \times(n-1)$ orthonormal column matrix and satisfies $v_{1}^{\dagger} B=0_{1, n-1}$; and also denote $V=\left[v_{1} \mid C\right]$. Then, we can easily show

$$
G_{1}^{\dagger} V=\left[\begin{array}{c}
v_{1}^{\dagger} \\
B^{\dagger}
\end{array}\right]\left[\begin{array}{ll}
v_{1} & C
\end{array}\right]=\left[\begin{array}{cc}
v_{1}^{\dagger} v_{1} & v_{1}^{\dagger} C \\
B^{\dagger} v_{1} & B^{\dagger} C
\end{array}\right]=\left[\begin{array}{cc}
1 & 0 \\
0 & V^{(2)}
\end{array}\right] .
$$

Since $\left(G_{1}^{\dagger} V\right)$ is an orthonormal column matrix, the right hand side of (10) must satisfy

$$
\left[\begin{array}{cc}
1 & 0 \\
0 & V^{(2)}
\end{array}\right]^{\dagger}\left[\begin{array}{cc}
1 & 0 \\
0 & V^{(2)}
\end{array}\right]=\left[\begin{array}{cc}
1 & 0 \\
0 & \left(V^{(2)}\right)^{\dagger} V^{(2)}
\end{array}\right]=I .
$$

Therefore, $V^{(2)}$ is an orthonormal column matrix.

The unitary matrix $G_{1}$ can be obtained in various ways, e.g., using Givens rotations and Householder reflections. One method using real Givens rotations is introduced in [6].

We can recursively apply the above procedure to the smaller orthonormal column matrix. That is, consider a $(t-1) \times(t-1)$ unitary matrix $\tilde{G}_{2}$ whose first column is the first column of $V^{(2)}$. Then, again by Lemma 1 ,

$$
\tilde{G}_{2}^{\dagger} V^{(2)}=\left[\begin{array}{cc}
1 & 0 \\
0 & V^{(3)}
\end{array}\right]
$$

where $V^{(3)}$ is a $(t-2) \times(n-2)$ orthonormal column matrix. This new step can be added in the matrix notation of (10).

$$
G_{2}^{\dagger} G_{1}^{\dagger} V=\left[\begin{array}{cc}
I_{2} & 0 \\
0 & V^{(3)}
\end{array}\right] \text {, where } G_{2}=\left[\begin{array}{cc}
1 & 0 \\
0 & \tilde{G}_{2}
\end{array}\right] .
$$

Let us denote $V^{(1)}=V$ and the first column of $V^{(i)}$ by $q_{i}$ for $i=1, \ldots, n$. An important observation is that $G_{i}$ is a function only depending on $q_{i}$. Therefore, after continuing the above procedure, finally the original orthonormal column matrix $V$ is factored as

$$
V=G_{1}\left(q_{1}\right) G_{2}\left(q_{2}\right) \cdots G_{n}\left(q_{n}\right) \tilde{I} .
$$

where the functional dependency of $G_{i}$ is explicitly indicated and $\tilde{I}=\left[I_{n}, 0_{n, t-n}\right]^{T}$. With this factorization, we see that a $t \times n$ orthonormal column matrix $V$ can be uniquely represented by a set of unit-norm vectors, $q_{1} \in \mathcal{S}_{t}, q_{2} \in \mathcal{S}_{t-1}$, $\ldots, q_{n} \in \mathcal{S}_{t-n+1}$.

\section{B. Statistical Properties}

In this section, we provide a key Theorem to show the statistical properties of the unit-norm vectors $q_{i}$ without proof (due to space limitations). A related definition is the complex Stiefel manifold, denoted by

$$
\mathcal{V}_{n, t}=\left\{V(t \times n): V^{\dagger} V=I_{n}\right\}
$$


TABLE I

THE QUANTIZATION METHOD FOR MIMO CHANNELS

\begin{tabular}{l|c|c}
\hline Parameterize at receiver & Quantize & Reconstruct at transmitter \\
\hline$v_{1}=q_{1}$ & $\hat{q}_{1}=\mathcal{Q}_{1}\left(q_{1}\right)$ & $\hat{v}_{1}=\hat{q}_{1}$ \\
$G_{1}^{\dagger} v_{2}=\left[\begin{array}{c}0 \\
q_{2}\end{array}\right]$ & $\hat{q}_{2}=\mathcal{Q}_{2}\left(q_{2}\right)$ & $\hat{v}_{2}=\hat{G}_{1}\left[\begin{array}{c}0 \\
\hat{q}_{2}\end{array}\right]$ \\
$\vdots$ & $\vdots$ & $\vdots$ \\
$G_{n-1}^{\dagger} \cdots G_{1}^{\dagger} v_{n}=\left[\begin{array}{c}0 \\
q_{n}\end{array}\right]$ & $\hat{q}_{n}=\mathcal{Q}_{n}\left(q_{n}\right)$ & $\hat{v}_{n}=\hat{G}_{1} \cdots \hat{G}_{n-1}\left[\begin{array}{c}0 \\
\hat{q}_{n}\end{array}\right]$ \\
\hline
\end{tabular}

The following Theorem describes statistical properties involved in the parameterization of Lemma 1.

Theorem 1: Consider the first step of the parameterization in Section IV-A for $t \times n$ random orthonormal column matrix $V$ uniformly distributed over $\mathcal{V}_{n, t}$.

$$
G_{1}^{\dagger} V=\left[\begin{array}{cc}
1 & 0 \\
0 & V^{(2)}
\end{array}\right]
$$

Then, the matrix $V^{(2)}$ is uniformly distributed over $\mathcal{V}_{n-1, t-1}$ and is independent of $v_{1}$. That is, the conditional joint density function of $V^{(2)}$ for given $v_{1}$ is given by

$$
p\left(V^{(2)} \mid v_{1}\right)=1 / \operatorname{Vol}\left(\mathcal{V}_{n-1, t-1}\right), \forall V^{(2)} \in \mathcal{V}_{n-1, t-1} .
$$

By applying the above Theorem recursively, we can arrive at the desired result stated in 2).

\section{The Quantization Method}

The basic idea of the quantization method for MIMO spatial information is to quantize unit-norm vectors $\left\{q_{i}\right\}$ independently using a set of codebooks $\left\{\mathcal{C}_{i}\right\}$. The codbook $\mathcal{C}_{i}$ is designed for random unit-norm vector in $\mathcal{S}_{t-i+1}$ with the MSIP criterion. The independence of the unit-norm vectors $\left\{q_{i}\right\}$ indicates that the overall loss is minimal compared to joint quantization of $q_{i}$. For example, a MIMO channel with $t=4$ and $r=2$ has a $4 \times 2$ random orthonormal column matrix $V$ for feedback. Using the parameterization method, we extract two unit-norm vectors, $q_{1} \in \mathbb{C}^{4}$ and $q_{2} \in \mathbb{C}^{3}$. Then, we quantize the two unit-norm vectors separately using two codebooks $\mathcal{C}_{1}$ and $\mathcal{C}_{2}$, which generally have different codebook sizes. Table I summarizes the overall procedure. Equivalent vector-wise notations are used since we can see the relations between $\left\{v_{i}\right\}$ and $\left\{q_{i}\right\}$ more clearly. Generally, the parameterization, the quantization, and the reconstruction procedures are described as

$$
\begin{gathered}
G_{i-1}^{\dagger} \cdots G_{1}^{\dagger} v_{i}=\left[\begin{array}{c}
0 \\
q_{i}
\end{array}\right], \\
\hat{q}_{i}=\mathcal{Q}_{i}\left(q_{i}\right), \\
\hat{v}_{i}=\hat{G}_{1} \cdots \hat{G}_{i-1}\left[\begin{array}{c}
0 \\
\hat{q}_{i}
\end{array}\right]
\end{gathered}
$$

for $i=1, \ldots, n$, where $\mathcal{Q}_{i}(\cdot)$ is quantization over $\mathcal{C}_{i}$ for $(t-i+1)$-dimensional random unit-norm vector, and $\hat{G}_{i}$ is the unitary matrix generated from the quantized unit-norm vector $\hat{q}_{i}$ in the same manner as in generating $G_{i}$ from $q_{i}$. The quantized version of $V$ can be also calculated as in (13)

$$
\hat{V}=\hat{G}_{1}\left(\hat{q}_{1}\right) \hat{G}_{2}\left(\hat{q}_{2}\right) \cdots \hat{G}_{n}\left(\hat{q}_{n}\right) \tilde{I} .
$$

Note that the reconstructed matrix $\hat{V}$ has same geometrical structure as the original $V$.

In quantizing the unit-norm vectors $q_{i}$ for given codebooks $\mathcal{C}_{i}$, we consider the following encoding schemes with different levels of complexity.

Encoding Scheme A: Encode sequentially $i=1, \ldots, n$ as

$$
\hat{q}_{i}=\arg \max _{\hat{q} \in \mathcal{C}_{i}}\left|\left\langle q_{i}, \hat{q}\right\rangle\right| .
$$

This simple scheme is expected to work well when the number of bits $B$ is large (high-rate quantization). However, when $B$ is small, we have some error propagation problem. That is, since the transmitter knows only quantized versions $\left\{\hat{q}_{i}\right\}$, the reconstructed $\hat{v}_{i}$ is affected by quantization errors in $\hat{q}_{1}, \ldots, \hat{q}_{i}$. In other words, the quantization errors in the previous vectors will accumulate. To alleviate this problem, we consider a new encoding scheme. Here, we consider maximizing $\left|\left\langle v_{i}, \hat{v}_{i}\right\rangle\right|$ after the vector is reconstructed.

Encoding Scheme B: Encode sequentially $i=1, \ldots, n$ as

$$
\hat{q}_{i}=\arg \max _{\hat{q} \in \mathcal{C}_{i}}\left|\left\langle v_{i}, \hat{v}\right\rangle\right|=\arg \max _{\hat{q} \in \mathcal{C}_{i}}\left|\left\langle\tilde{q}_{i}, \hat{q}\right\rangle\right| .
$$

where $\hat{v}=\hat{G}_{1} \cdots \hat{G}_{i-1}\left[\begin{array}{ll}0 & \hat{q}\end{array}\right]^{T}$, and $\tilde{q}_{i}$ is related with $v_{i}$ as

$$
\hat{G}_{i-1}^{\dagger} \cdots \hat{G}_{1}^{\dagger} v_{i}=\left[\begin{array}{c}
a \\
\tilde{q}_{i}
\end{array}\right] \text {, for some } a \in \mathbb{C}^{i-1} \text {. }
$$

Encoding Scheme $C$ : Consider the composite codebook $\mathcal{C}$ for $V$ from $\mathcal{C}_{1}, \mathcal{C}_{2}, \ldots \mathcal{C}_{n}$, i.e., $|\mathcal{C}|=\left|\mathcal{C}_{1}\right| \times\left|\mathcal{C}_{2}\right| \times \cdots \times\left|\mathcal{C}_{n}\right|$. Encode to maximize the mutual information as

$$
\hat{V}=\arg \max _{\hat{W} \in \mathcal{C}} \max _{\gamma} \log \operatorname{det}\left[I+H \hat{W} \Phi(\gamma) \hat{W}^{\dagger} H\right] .
$$

\section{Bit Allocation and Numerical Results}

For simulations, we considered MIMO channels with i.i.d. $\mathcal{C N}(0,1)$ entries with $t=4$ and $r=2$. To quantize a $4 \times 2$ orthonormal column matrix $V$, the total number of quantization bits is fixed to $B=8$ bits. Here a bit allocation problem occurs. There are many possible ways of allocating the fixed number of bits in quantizing the unit-norm vectors, $q_{1} \in \mathcal{S}_{4}$ and $q_{2} \in \mathcal{S}_{3}$, and power allocation $\gamma$. We considered the following cases in simulations:

$$
\left[B_{1} B_{2} ; B_{\gamma}\right]=[80 ; 0],[51 ; 2],[42 ; 2],[33 ; 2],[24 ; 2],[00 ; 8]
$$

where $B_{i}$ is the number of bits allocated to $q_{i}$ and $B_{\gamma}$ for $\gamma$ with $B_{1}+B_{2}+B_{\gamma}=B$. For example, the first case $[80 ; 0]$ means that the whole bits are used to quantize only $q_{1}$ and do not feed back $q_{2}$ (quantized maximal ratio transmission). The MSE VQ design procedure is used to design the codebooks for power allocation.

We present here results only with the Encoding Scheme B due to space limitations. Fig. 3 shows the average capacities 
with different bit allocations. For ease of comparison, all the capacities have been normalized to the capacity with the complete CSIT $\left(C_{\text {Full }}\right)$. We can see that the optimum bit allocation is dependent on what transmit power the system is operating at. At low power region, the optimum way is to quantize only first unit-norm vector $q_{1}$, i.e., $\left[B_{1} B_{2} ; B_{\gamma}\right]=$ $[80 ; 0]$. As power increases, we need to change bit allocation to $[51 ; 2]$ and $[42 ; 2]$. And, in the high power region, the optimum bit allocation is $[33 ; 2]$.

Another way of bit allocation is to use all bits to quantize spatial information only, and employ equal power allocation in transmitting the data. Simulation results (not shown here) shows that it provides a better performance in high SNR region. This is because when transmit power is high, the optimum power allocation is equal power allocation in the most case, therefore, it is a waste to quantize the almost predetermined information.

\section{CONCLusion}

We investigated quantization of channel information for multiple antenna communication systems. For MISO systems, we proposed a new design criterion, namely, maximizing mean squared inner-product (MSIP), and developed the corresponding quantizer design algorithm for random unitnorm vector with arbitrary dimension and codebook size. For MIMO channels, it was shown that an orthonormal column matrix is uniquely represented by a set of unit-norm vectors with different dimensions, and we showed related statistical properties, especially, independence between the unit-norm vectors. The unit-norm vectors are quantized independently using codebooks designed in the MSIP criterion. Results show that the quantization bit allocation over multiple spatial channels has a critical effect on the performance, and that the optimum bit allocation depends on the operating transmit power of the system. We have also investigated joint quantization schemes for multiple directional vectors, which will be reported in a future publication.

\section{REFERENCES}

[1] I. E. Telatar, "Capacity of multi-antenna Gaussian channels," AT\&T Bell Labs Tech. Memo., 1995.

[2] A. Narula, M. J. Lopez, M. D. Trott, and G. W. Wornell, "Efficient use of side information in multiple-antenna data transmission over fading channels," IEEE J. Select. Areas Commun., vol. 16, pp. 1423 - 1436, Oct. 1998.

[3] K. K. Mukkavilli, A. Sabharwal, E. Erkip, and B. Aazhang, "On beamforming with finite rate feedback in multiple antenna systems," IEEE Trans. Inform. Theory, vol. 49, no. 10, pp. 2562 - 2579, Oct. 2003.

[4] D. J. Love, R. Heath, Jr., and T. Strohmer, "Grassmannian beamforming for multiple-input multiple-output wireless systems," IEEE Trans. Inform. Theory, vol. 49, no. 10, pp. 2735 - 2747, Oct. 2003.

[5] D. J. Love and R. Heath, Jr., "Limited feedback precoding for spatial multiplexing systems," in Proc. IEEE Globecom 2003, San Francisco, CA, Dec. 2003.

[6] J. C. Roh and B. D. Rao, "An efficient feedback method for MIMO systems with slowly time-varying channels," in Proc. IEEE WCNC 2004, Atlanta, Georgia, USA, Mar. 2004.

[7] C. Murthy, J. C. Roh, and B. D. Rao, "Optimality of extended maximum ratio transmission," in 6th Baiona Workshop on Signal Processing in Communications, Baiona, Spain, Sept. 2003.
[8] J. C. Roh and B. D. Rao, "Multiple antenna channels with partial channel state information at the transmitter," IEEE Trans. Wireless Commun., Mar. 2004.

[9] A. Gersho and R. M. Gray, Vector Quantization and Signal Compression. Kluwer Academic, 1992.

[10] J. C. Roh and B. D. Rao, "Beamforming vector quantization for multiple antenna systems with finite rate feedback," in preparation.

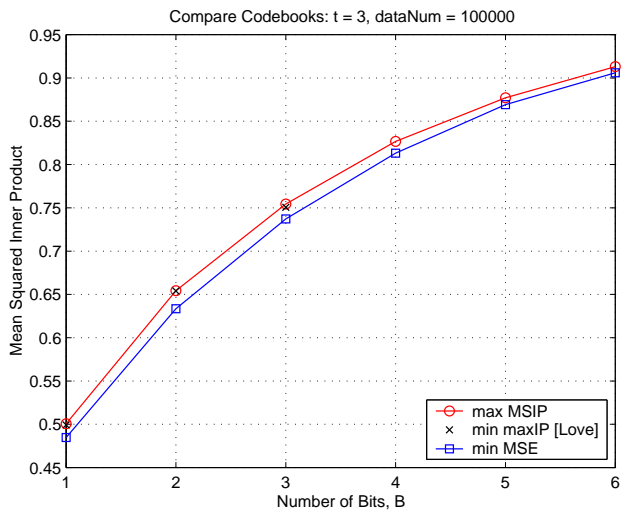

Fig. 1. Examples of quantizer design with the MSIP criterion and comparison with other design criteria $\left(t=3\right.$ and $\left.N=2^{B}, B=1,2 \ldots, 6\right)$.

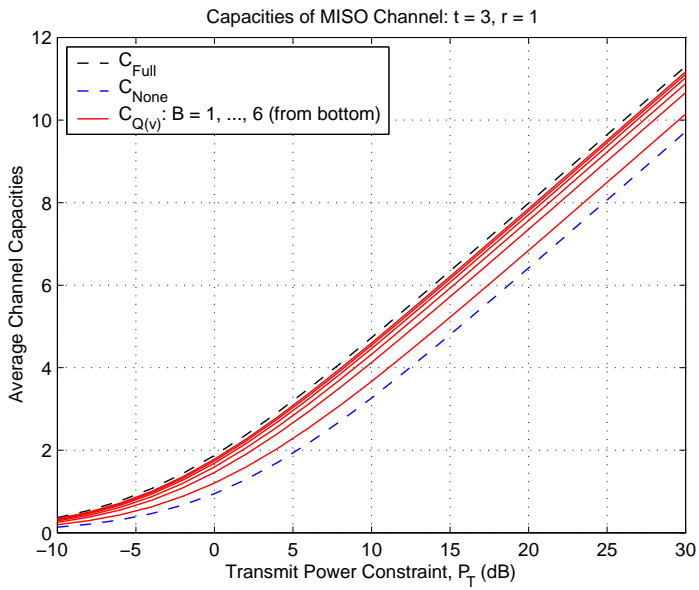

Fig. 2. Average capacities of MISO channel with quantized feedback $(t=3$ and $\left.N=2^{B}, B=1,2 \ldots, 6\right)$

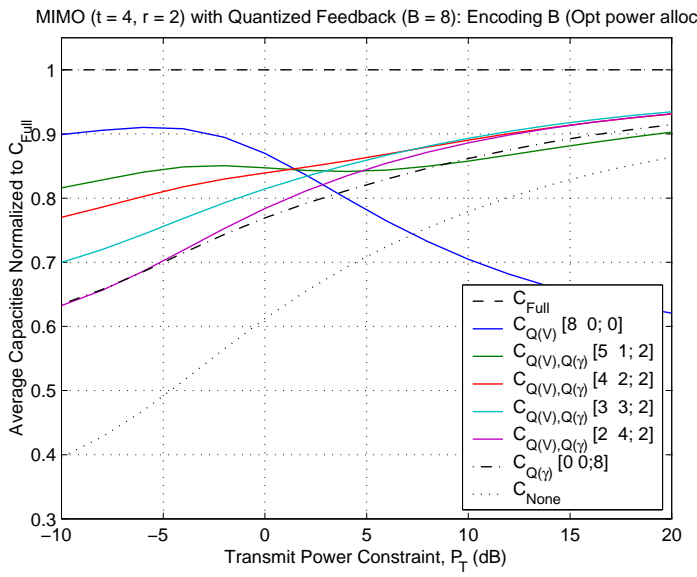

Fig. 3. Normalized average capacities of MIMO channel with quantized feedback and different bit allocations $(t=4, r=2$ and $B=8)$. 